\title{
Current Status of International Airborne Platform Data and Instrument Interface Standards
}

\author{
Matt Freer ${ }^{1}$, Chris Webster ${ }^{2}$, Larry Freundinger ${ }^{3}$ \\ ${ }^{1}$ Droplet Measurement Technologies, United States of America; ${ }^{2}$ NCAR RAF, \\ United States of America; ${ }^{3}$ NASA Dryden, United States of America
}

\section{Commission VI, WG VI/4}

\begin{abstract}
Commission I of the International Society for Photogrammetric and Remote Sensing formed working group $I / 1$ for the purpose of standardizing airborne platform interfaces. The primary mission of this working group is to promote the standardization of instrument interfaces, data formats, and supporting infrastructures; and to facilitate more efficient, flexible, and cost-effective international science flight operations. Within WGI/1 are a number of focused subgroups. This paper addresses the efforts of some of these subgroups having interdependent and overlapping interests; including the development of standard software interfaces for sensors, standardized approaches to management of information over potentially intermittent wireless data links, the development of standardized processing algorithms, and data archival format standards. The data in this report reflect the outcome of work-to-date in the current subgroups.
\end{abstract}

Keywords: environment, facilities, GIS, database, internet/web, technology, atmosphere, engineering, airborne science platform systems

\section{INTRODUCTION}

Research aircraft are an important contributing layer of the GEOSS (Global Earth Observation System of Systems) era. Commission 1 of the International Society for Photogrammetric and Remote Sensing (ISPRS) working group I/1 (WGI/1) facilitates the emergence of GEOSS by promoting standardization of instrument interfaces, data formats and supporting infrastructures, thereby making airborne science more efficient, productive and useful. The ISPRS WGI/1 TOR2311 team is a combination of three former subgroups - TORs 2, 3 and $11-$ which are focused on developing standards for instrument integration, data distribution, and data processing associated with airborne science operations, respectively. The combination of these subgroups will encourage collaboration on these overlapping issues, and permit the exchange of ideas between experts within the subgroups.

The TOR2311 team held its first meeting during the International Conference on Airborne Research (ICARE) 2010 in Toulouse, France. Since this time, the group has endeavored to survey the current state of standards, and apply these wherever possible. This paper provides a summary of the ongoing efforts of this group to develop standards in the key areas of airborne payload networks, interactive communications such as satellite data links to and from those payload networks, and common data processing tools to facilitate productive intercomparisons amongst different instruments and aircraft.

\section{PAYLOAD NETWORKS}

Aircraft payload networks provide a critical function in modern airborne research by allowing intercommunication among instruments, aircraft data sources, and other services. Accurate time and vehicle position data distribution, for example, is a service that adds value and reduces instrument measurement errors. While the establishment of onboard instrumentation payload networks has become commonplace, a goal exists to facilitate and promote common sets of best practices for the payload networks on research aircraft.

The TOR2311 team has identified several areas that can benefit from such standardization. Currently, several de facto standards previously developed by the US-based Interagency Working Group for Airborne Data and Telemetry Systems (IWGADTS) have been successfully implemented in the US airborne science community. With these in place, international community can build on the prior work, and apply these standards more broadly.

For example, a common approach for intra-aircraft low-rate navigation data is needed. The so-called "IWG1" packet, developed by IWGADTS, defines a core set of measurands to be broadcast in regular fashion on the payload network. The current time, vehicle location and attitude, and a total of 31 measurands are defined. Distribution nominally occurs at one-second intervals using user datagram protocol (UDP). To take the standardization effort a step further, the IWG1 IWGADTS aircraft housekeeping message was officially registered as port number 7071 with the Internet Assigned Numbers Authority (IANA).

Topics remaining to be addressed include recommendations to reduce timing and spatial errors of instruments on the network. Standardization of documentation across platforms for describing sources of error would benefit researchers. Implementation of advanced network timing technologies and more accurate positioning such as Avionics Full Duplex Ethernet (AFDX, ARINC-664), Precision Timing Protocol (PTP, IEEE-1588), and differential global positioning system (GPS) are topics which still require standards definition in future TOR2311 meetings. 


\section{DATA LINKS}

While the payload network on the vehicle establishes the environment that enables progress, extending communications to and from the vehicle is a force multiplier. A major component of the GEOSS vision is real-time communication between the observation platforms and the decision-makers and end users. In the airborne science community, several aircraft operators have deployed satellite communication technologies to share their data in real time, and, in some cases, provided remote users with control over on-board instrument systems. Other aircraft have implemented air-to-ground data links. While some of these airborne-communication link technologies are operationally mature, others still require a fair level of technical expertise to use effectively. To meet the goals of GEOSS, the TOR2311 team aims to facilitate implementation of data-link connectivity on airborne platforms of all sizes.

The core of this activity will be the creation of documentation and recommendations covering all aspects of data-link setup. A survey will be performed on the current use of data links within airborne research to gauge bandwidth requirements, explore problems with current scenarios, and analyze reliability and cost issues. Based on the survey results, the TOR2311 team will make recommendations to the community for development of a network system, including details on installation, architecture, security and integrity, coverage, and strategic planning. There are also possibilities for experts within the TOR2311 team to aid operators with implementation of a data-link system.

The team envisions three distinct phases of data-link adoption, moving from the simplest to the most complex. Situational awareness in the form of automated flight following, which includes platform position, heading, and status, is the simplest to deploy, requiring only a small amount of bandwidth, but is invaluable for ground-based decision-makers for coordination of flights. The next level of complexity is instrument status and control, which requires more bandwidth and experiment-specific customization. This level can greatly benefit operators by allowing experts to remotely interface with instruments and payloads. The final phase is full-fledged data transfer, which allows all platform data to be accessed in real time from other platforms or from ground-based personnel. Satisfying this level of expectation in bandwidth, usability, and affordability is currently beyond the state of art except in constrained usage scenarios. Our recommendations will guide operators in adoption of all three phases of data-link adoption.

\section{PROCESSING TOOLS}

Well-developed processing tools form an important component of airborne research. A properly-developed software suite can provide researchers with the ability to analyze and view data from many platforms and instruments. Such software can greatly aid comparison between different types of aircraft and instruments. Equally important in analysis of airborne research data is the format of the data and its metadata. Standardized data formats with descriptive metadata can reduce usage errors and facilitate automatic data processing.

To meet these aircraft data processing needs, the TOR2311 team has adopted an open-source data-processing library and toolbox called EGADS (EUFAR General Airborne Data-processing Software), developed by the Standards and Protocols Networking Activity within the EUFAR project. This toolbox is a Python-based software framework which includes a library of algorithms contributed by experts in the airborne scientific community. Users interact with these algorithms via command-line or simple Python scripts. Further development will include visualization packages for plotting data and creating processing chains.

In parallel with development of a toolbox, the team has developed a common data and metadata convention using existing conventions, giving momentum to community adoption. Both ASCII and binary formats are supported by these conventions. The preferred ASCII format is NASA Ames and the preferred binary format is NetCDF/HDF. Overall, the binary format is preferred, as these formats have good support for descriptive metadata. In addition to these binary formats, the team has developed recommendations for data discovery, that is, metadata that helps direct users or software to find the data they need within large, complex data sets. These data discovery elements define common aircraft platform data parameter tags (latitude, longitude, altitude, etc.), thereby simplifying the task of automating data file input and processing.

In a larger context, processing tools evolve toward applications and services on the ground. Some of these tools will be purely post-test while others will support real-time decision-making. Several European and US research platforms had gathered for the ICARE conference; TOR2311 team members used this opportunity to informally experiment with Open Source DataTurbine (OSDT), an open source middleware caching and distribution infrastructure with real-time processing tools. OSDT is used in a variety of environmental monitoring and sensor web applications, including the NASA Airborne Science Program.

OSDT allowed distribution and processing of IWG1 packets on the ground, using them to drive Web-oriented displays that gave ground personnel situational awareness via the data link on both the SAFIRE Falcon 20 and the NCAR C- 
130. Thus, the international working group was quick to tie payload networks, data links, and processing tools together in an end-to-end scenario that provided a glimpse of the TOR2311 team vision.

\section{CONCLUSION}

The ISPRS WGI/1 TOR2311 team has created a set of de facto standards and recommendations for airborne payload networks, data links and processing tools that will facilitate the emergence of the Global Earth Observation System of Systems. These standards will facilitate the exchange of instruments and data systems between platforms; enable data to be seamlessly shared in real time during projects to aircraft operators, other platforms and ground-based users and decision-makers; and help remove barriers to postproject data sharing and intercomparison.

Dissemination of the recommendations proposed by the TOR2311 team are available both through the Interagency Working Group for Airborne Data and Telemetry Systems (IWGADTS) website at https://www.eol.ucar.edu/iwgadts/ and through the European Facility for Airborne Research (EUFAR) website at http://eufar.net/ under the Standards and Protocols activity section as well as the Good Practices resource. 